\title{
REGISTRO DE LA PARINA CHICA (Phoenicoparrus jamesi Sclater, 1886) EN LA RESERVA NACIONAL DE PARACAS, PERÚ
}

\section{RECORD OF THE PUNA FLAMINGO (Phoenicoparrus jamesi Sclater, 1886) IN THE PARACAS NATIONAL REVERVA, PERU}

\author{
Julissa Arenas ${ }^{1}$ y Francesca Montero ${ }^{12}$
}

\begin{abstract}
Resumen
Se reportó la presencia de la "parina chica" o "flamenco de James" Phoenicoparrus jamesi en la Bahía de Paracas dentro de la Reserva Nacional de Paracas (RNP). Esta especie no se encuentra actualmente incluida en su lista de aves. Se debe tener presente que esta especie se encuentra catalogada bajo la categoría de Casi Amenazada por la IUCN, además de considerarse endémica de las lagunas saladas altoandinas de Argentina, Bolivia, Chile y Perú.
\end{abstract}

Palabras clave: casi amenazada, endémica de lagunas altoandinas, parina chica, Phoenicoparrus jamesi, Reserva Nacional de Paracas, registro nuevo.

\begin{abstract}
The presence of the James's Flamingo also known as the Puna Flamingo (Phoenicoparrus jamesi) was reported in the Bay of Paracas within the limits of the Paracas National Reserve. This species is currently not included in the Reserve's list of birds. It must be considered that this species in listed by the IUCN under the category of Near Threatened, and is also considered as an endemic species of the Andean salt lakes of Argentina, Bolivia, Chile and Peru.

Key words: endemic of Andean lakes, Near Threatened, new record, Paracas National Reserve, Phoenicoparrus jamesi, Puna flamingo.
\end{abstract}

\section{Introducción.}

En el Perú habitan tres especies de parihuanas, de las cuales el "flamenco de James" Phoenicoparrus jamesi junto con la "parina grande" Phoenicoparrus andinus son las menos abundantes y con distribución más restringida, siendo consideradas las dos especies de flamencos más raras del mundo (Caziani et al., 2007).

El flamenco de James es una especie gregaria que habita ambientes de agua salina y anida en colonias, viaja largas distancias entre las zonas de reproducción y alimentación (Caziani et al., 2007); se alimenta principalmente de diatomeas; reproduciéndose en verano en las lagunas altoandinas, mientras que en invierno, muchos individuos viajan a humedales de menor altitud en las llanuras centrales de Argentina y la costa peruana (Parada et al., 1990).

$P$. jamesi es la especie más pequeña y presenta menor coloración negra en el pico (Schulenberg et al., 2007), abarcando sólo el primer tercio de la punta, a diferencia de Phoenicopterus chilensis y Phoenicoparrus andinus donde el color negro llega hasta la mitad del pico (Aves de Chile, 2014). Los tarsos de los adultos son completamente rojos (Figura 2) y carecen del cuarto dedo o "pulgar" (Aves de Chile, 2014); mientras que el plumaje del juvenil es más apagado, pero identificable por tamaño, con menor coloración negra en el pico (Schulenberg et al., 2007) y con los tarsos de color negro (Figura 3).

En el Perú es considerada visitante no reproductivo en el sur (especie migratoria austral) por encima de los $4000 \mathrm{msnm}$; divagante y muy rara en la Costa y en la Amazonía (Schulenberg et al., 2007). Los lagos altiplánicos como Salinas (Arequipa), Loriscota (Puno), Suches (Perú-Bolivia), Vilacota (Tacna), Vizcachas (Moquegua) y Blanca (Tacna) son conocidos como importantes territorios para flamencos y están amenazados por la minería, proyectos de agua potable, irrigación y destrucción del hábitat; siendo Salinas el único protegido como ANP (Ricalde, 2003).

En años anteriores $P$. jamesi ha sido registrada en lugares como el Santuario Nacional Lagunas de Mejía (Hogsas et al., 2010), en cuya lista de especies se encuentra como especie ocasional del área (INRENA, 2000). Así mismo, entre abril y julio del 2012 se reportaron individuos de esta especie en los humedales de Ite, Tacna (Vizcarra \& Vicetti, 2013), siendo este el registro más meridional de esta especie en la costa peruana $\left(17^{\circ} \mathrm{S}\right)$.

Para la RNP la especialista Patricia Saravia reportó 3 registros para Mayo 2012 (eBrid, 2013), sin embargo, esta especie no está incluida en la lista de aves de la Reserva Nacional de Paracas, que incluye 
solo una especie de flamenco, el "flamenco chileno" Phoenicopterus chilensis.

\section{Materiales y métodos.}

La evaluación de aves se realizó mensualmente por el borde de orilla en toda la Bahía de Paracas, desde las 06:00 hasta las 11:30 horas, usándose el método de conteo directo por transectos (Ralph et al., 1996; Bibby et al., 1998; Botero y Arbeláez, 2005) con binoculares, libreta de campo, GPS y cámara fotográfica.

La zona evaluada fue la Bahía de Paracas ubicada dentro de la Reserva Nacional de Paracas (RNP), específicamente en playa La Aguada entre las coordenadas 13 $51^{\prime} 41^{\prime}$ 'S $76^{\circ} 16^{\prime} 68^{\prime \prime} \mathrm{O}$ y $13^{\circ} 52^{\prime} 00^{\prime \prime} \mathrm{S} \quad-$ $76^{\circ} 15^{\prime} 46^{\prime \prime} \mathrm{O}$ (Figura 1).

La playa La Aguada es una zona comprendida desde la orilla de mar hasta la carretera al Puerto San Martín; limita al Este con la zona de recuperación de Santo Domingo y al Oeste con la zona de protección estricta de la Bahía de Paracas. Está considerada como Zona Silvestre, es decir es un área que ha sufrido poca o nula intervención humana, en la que predomina el carácter silvestre, es posible realizar actividades de investigación científica, educación y

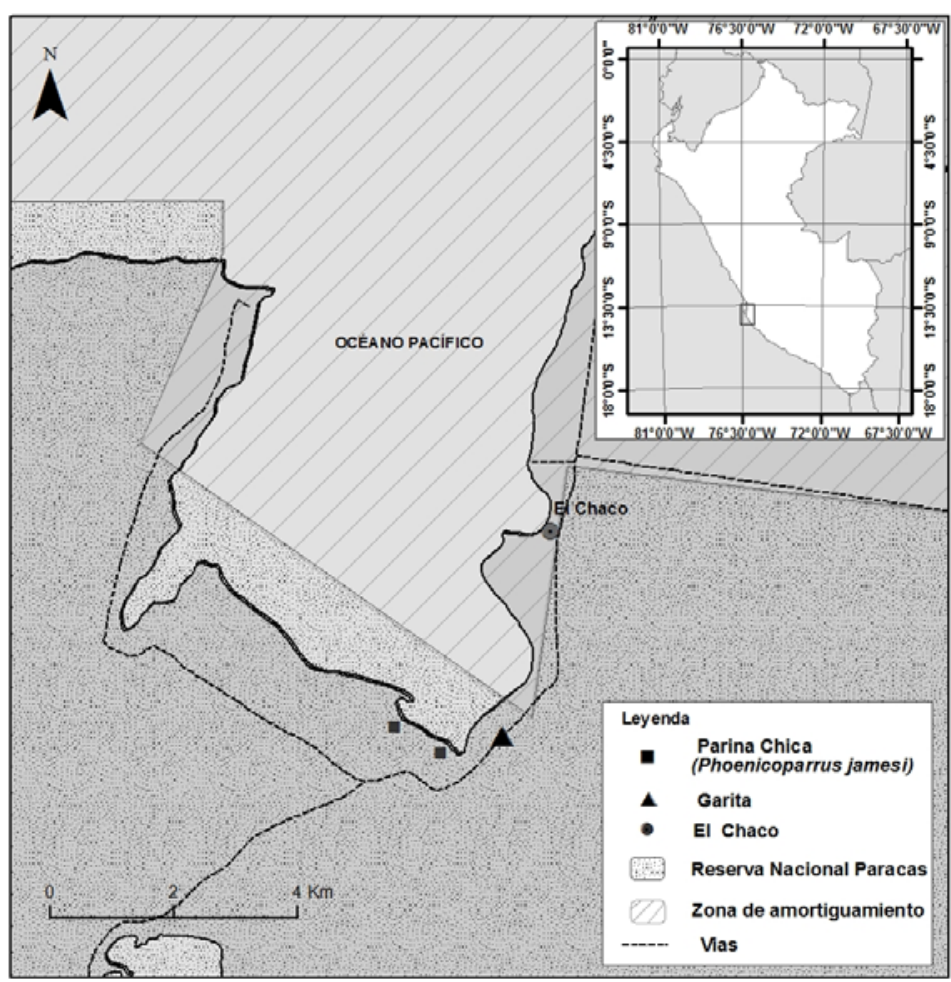

Figura 1. Mapa de Ubicación de los registros de "parina chica" $P$. jamesi dentro de la Reserva Nacional de Paracas recreación sin infraestructura permanente ni vehículos motorizados (INRENA, 2002).

\section{Resultados y discusión.}

Entre los meses de Julio y Diciembre 2012, como parte de la evaluación de aves del Monitoreo Biótico y Abiótico llevada a cabo por la consultora ERM Perú S.A., se ha registrado la presencia del $P$. jamesi en la Bahía de Paracas, lugar que se caracteriza por sus aguas tranquilas, sin oleaje (Arenas, 1993) y por presentar sustrato fangoso de poca profundidad y poco deslizamiento de corrientes y oleajes (PNUMA y CONAM, 2007).

En total se obtuvo 15 registros de esta especie; entre adultos y juveniles: 2 juveniles y 1 adulto en el mes de Julio; 2 juveniles y 9 adultos en el mes de agosto; ningún individuo en el mes de setiembre, 1 individuo juvenil en el mes de Octubre y ningún registro en los meses de Noviembre y Diciembre (Figuras 2 y 3 ).

En la Bahía de Paracas la presencia de esta especie fue reportada en mayo (eBird, 2013) y entre Julio y Octubre, mientras que en los Humedales de Ite entre los meses de Abril y Julio, con reportes en su mayoría de individuos juveniles (Vizcarra \& Vicetti, 2013), en contraposición con el mayor número de individuos adultos para la Bahía de Paracas. Estos meses corresponden a las épocas de otoño, invierno y primavera, obteniéndose para las dos zonas el mayor número de registros en las dos primeras épocas (otoño e invierno). 


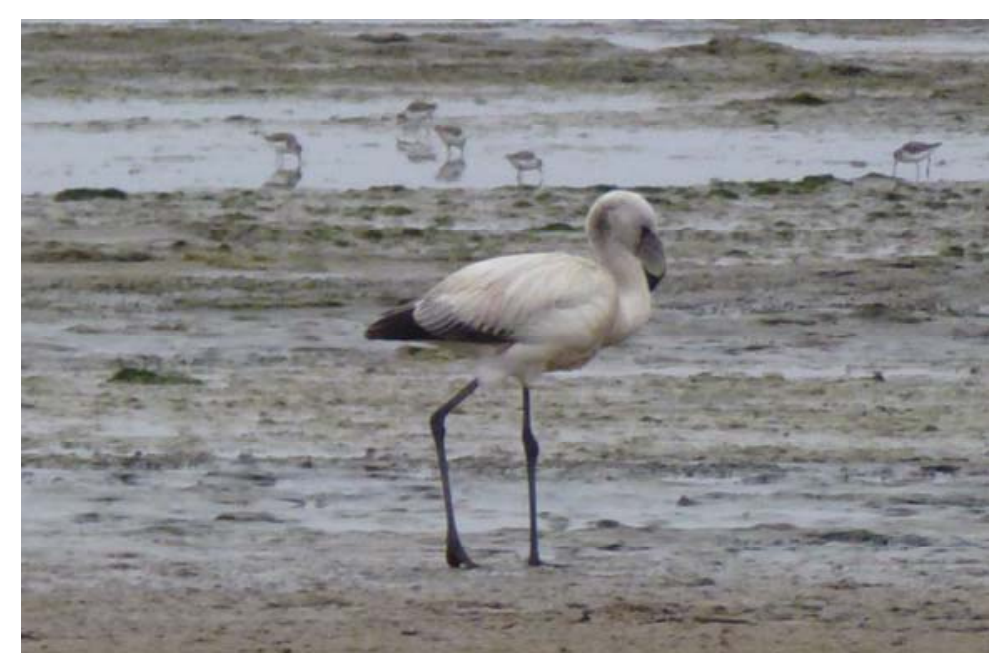

Figura 3. Individuo juvenil de $P$. jamesi en la playa La Aguada, RNP - Julio 2012. Se puede apreciar el plumaje más apagado, además de la carencia del cuarto dedo en las patas. Foto: Julissa Arenas.

Estos resultados confirmarían el movimiento migratorio de esta especie entre las zonas de reproducción y alimentación (Caziani et al., 2007); ya que se reproduce en verano en las lagunas altoandinas, y en invierno muchos individuos se desplazan hacia los humedales de menor altitud en las llanuras centrales de Argentina y la costa peruana (Parada et al., 1990). Estos individuos podrían provenir de la Laguna Colorada en Bolivia, ya que es la zona reproductiva con la mayor colonia de $P$. jamesi (Valqui et al., 2000) y la más cercana a la costa peruana.

La importancia del registro de $P$. jamesi en la Bahía de Paracas radica en el hecho de ser la observación de distribución más septentrional para esta especie $\left(13^{\circ} \mathrm{S}\right)$ reforzando la idea de incluir la especie en el listado de Aves de la Reserva Nacional de Paracas como especie divagante. Información que consideramos debe ser relevante por encontrarse en la categoría de Casi Amenazada (NT) por la Unión Internacional para la Conservación de la Naturaleza (IUCN, 2012), y ser considerada especie endémica de las lagunas saladas altoandinas de Argentina, Bolivia, Chile y Perú (Valqui et al., 2000; Caziani et al., 2007).

\section{Conclusiones.}

Se refuerza la idea de ampliación en el rango de distribución norte y de añadir el nuevo registro de "parina chica" o "flamenco de James" P. jamesi en el listado de aves de la Reserva Nacional de Paracas por lo tanto esta especie debería incluirse en la lista de aves del Plan Maestro de esta ANP como una especie con presencia ocasional en el área.

\section{Recomendaciones}

Continuar con los monitoreos mensuales de aves en el área de Bahía de la Reserva Nacional de Paracas pues es crucial e importante los aportes de nuevos registros.

\section{Agradecimientos.}

Agradecemos a Environmental Resources Management Perú (ERM), consultora encargada a la fecha del Monitoreo Biótico y Abiótico, en especial a Francisco Pinilla y Julio Arenas por el apoyo brindado para la realización de las evaluaciones mensuales. A la empresa Pluspetrol Perú Corporation S.A. por su compromiso con el Monitoreo y su apoyo en la publicación de este manuscrito. A la Reserva Nacional de Paracas por el permiso otorgado y el apoyo brindado a las autoras. A Manuel Plenge por sus aportes y sugerencias con respecto a los registros de $P$. jamesi para el Perú.

\section{Literatura citada.}

Arenas J. 1993. Paracas. Editorial Supergráfica E.I.R.L. Lima, Perú.

Aves de Chile Parina chica. 2014. $<$ http://www.avesdechile.cl/031.htm>. Consulta el 07/01/2014.

Bibby C., Jones, M. \& Marsden S. 1998. Bird surveys, Expedition Field Techniques. Royal Geographical Society.

Botero J. \& Arbeláez D. 2005. Métodos para estudiar las aves. Biocarta $\mathrm{N}^{\circ} 8$.

Caziani S., Rocha O., Rodriguez E., Romano M., Derlindati E., Talamo A., Ricalde D., Quiroga C., Contreras J., Valqui M. \& Sosa H. 2007. Seasonal Distribution, Abundance, and Nesting of Puna, Andean, and Chilean Flamingos. The Condor 109:276-287.

eBird. 2013. eBird: Una base de datos en línea para la abundancia y distribución de las aves. $<$ http://ebird.org/ebird/peru/GuideMe?cmd=quickPick\& speciesCode $=$ \&bMonth $=01 \&$ bYear $=1900$ \&eMonth $=12$ \&eYear $=2013 \&$ getLocations $=$ countries \&countries $=\mathrm{PE}$ \&reportType $=$ species $\&$ speciesCodes $=$ jamfla $\&$ continue $. \mathrm{x}=66 \&$ continue $. \mathrm{y}=10 \&$ continue $=$ Continue $>$. Consulta el $12 / 10 / 2013$

Hogsas T., Vizcarra J., Hidalgo N. \& Málaga E. 2010. Primeros registros documentados de Phoenicoparrus andinus en la costa sur del Perú. Cotinga 32: Shorts communications, $120-121$.

Instituto Nacional de Recursos Naturales (INRENA). 2000. Santuario Nacional Lagunas de Mejía. Plan Maestro 2000 - 2005.

Instituto Nacional de Recursos Naturales (INRENA). 2002. Reserva Nacional de Paracas. Plan Maestro 2003 2007.

IUCN. 2012. IUCN Red List of Threatened Species. Version 2012.2. <www.iucnredlist.org>. Consulta el 17/10/2012. 
Parada M., Rottmann J. \& Guerra C. 1990. Flamencos en el norte de Chile: distribución, abundancia y fluctuaciones estacionales, p. 54-66. I taller internacional de especialistas en flamencos sudamericanos. Corporación Nacional Forestal y Sociedad Zoológica de Nueva Cork, San Pedro de Atacama, Chile.

Programa de las Naciones Unidas para el Medio Ambiente (PNUMA) y Consejo Nacional del Ambiente (CONAM). 2007. Informe sobre el estado del Ambiente, GEO Bahía Paracas - Pisco. 164p.

Ralph J., Geupel G., Pyle P., Martin T., DeSante D. \& Borja, M. 1996. Manual de métodos de campo para el monitoreo de aves terrestres. General Technical Report PSW-GTR-159. Albany, CA: Pacific Southwest Research Station, Forest Service, U.S. Department of Agriculture, $46 \mathrm{p}$.

Resolución Jefatural № 001-2012-SERNANP-RNP. 2012. Permiso de Investigación.
Resolución Presidencial No 216-2009-SERNANP. 2009. Ampliación de Vigencia del Plan Maestro de la Reserva Nacional de Paracas.

Ricalde D. 2003. Conservación de flamencos en el altiplano peruano. Lyonia 4(1): 87-96.

Schulenberg S., Stotz T., Lane D. F., O'Neill J. P. \& Parker III T. A. 2007. Birds of Peru. Princeton University Press. 660p.

Valqui M., Caziani S., Rocha O. \& Rodriguez E. 2000. Abundance and distribution of the South American Altiplano Flamingos. Waterbirds: The International Journal of Waterbird Biology, Vol. 23, Special Publication 1: Conservation Biology of Flamingos, pp. 110-113.

Vizcarra J. \& Vicetti R. 2013. Primer registro documentado de la Parina chica (Phoenicopharrus jamesi) en los Humedales de Ite, costa sur del Perú. Boletín Informativo de la Unión de Ornitólogos del Perú (UNOP), 8(1): 21-26.

\footnotetext{
${ }^{1}$ Environmental Resources Management Perú S.A. (ERM). Av. Canaval y Moreyra 694 San Isidro. julissa145@hotmail.com,galia_mc@yahoo.es

${ }^{2}$ Universidad Nacional Agraria La Molina Facultad Maestría Conservación de Recursos Forestales. Av. La Molina s/n La Molina.
} 